\title{
Die MIST-Studie
}

\section{Migraine Intervention with STARFlex Technology}

Hintergrund und Problemstellung: Bei Patienten mit großem offenen Foramen ovale (PFO) wurden in ca. 50\% der Fälle auch schwere Migräneanfälle dokumentiert. In mehreren Beobachtungsstudien (Registern) konnte gezeigt werden, dass der mechanische Verschluss (mittels Okkludersystem) eines funktionellen PFO (d.h. mit konsekutivem Rechts-linksShunt) zu einer Verbesserung der Migränesymptomatik führt. Zugrunde gelegt wurde die (unbestätigte) Hypothese, dass es spezifische Substanzen gibt, die bei der Lungenpassage normalerweise „entgiftet“ werden, durch den Rechts-linksShunt jedoch direkt in das ZNS gelangen, wo sie die Migräne-

\begin{tabular}{|c|c|}
\hline Methodik & \\
\hline Studiendesign & $\begin{array}{l}\text { Prospektive, randomisierte, doppelblinde Multi- } \\
\text { centerstudie } \\
\text { Nachweis eines PF0 mittels transthorakaler } \\
\text { Echokardiographie } \\
\text { Vollnarkose bei allen Patienten und Inzision } \\
\text { der Leiste (auch in der Plazebogruppe) } \\
\text { Die Nachbeobachtung betrug } 6 \text { Monate. } \\
\text { 3-monatige postprozedurale Behandlung } \\
\text { mit ASS und Clopidogrel } \\
\text { Alle Patienten wurden nicht von den inter- } \\
\text { ventionellen Kardiologen, sondern von gegen- } \\
\text { über dem interventionellen Verfahren geblinde- } \\
\text { ten Neurologen monatlich nachverfolgt }\end{array}$ \\
\hline Primärer Endpunkt & $\begin{array}{l}\text { Vollständige Beschwerdefreiheit (bezüglich An- } \\
\text { fallshäufigkeit und -dauer) der Migräne }\end{array}$ \\
\hline Patientenauswahl & $\begin{array}{l}\text { Einschlusskriterien: } 18-60 \text { Jahre, }>1 \text { Jahr Mi- } \\
\text { gräne. Anfallshäufigkeit } \geq 5 \text { Tage/Monat, } \\
\geq 2 \text { Migränemedikamente erfolglos eingenommen } \\
\text { Ausschlusskriterien: Z.n. TIA/Schlaganfall, kar- } \\
\text { diale Kontraindikationen }\end{array}$ \\
\hline $\begin{array}{l}\text { Patientenzahl in den } \\
\text { randomisierten } \\
\text { Untergruppen }\end{array}$ & $\begin{array}{l}\text { Scheinprozedur } \\
\mathrm{n}=73\end{array}$ \\
\hline
\end{tabular}

Ergebnisse: $\left({ }^{*} p<0,05\right)$

\begin{tabular}{|c|c|c|}
\hline $\begin{array}{l}\text { Ausgangsdaten: } \\
\text { Patienten- } \\
\text { charakteristika }\end{array}$ & \multicolumn{2}{|c|}{$\begin{array}{l}\text { keine Unterschiede bezüglich Alter, Geschlecht, } \\
\text { Anfallshäufigkeit und -dauer der Migräne, der } \\
\text { PF0-Diameter war (von transthorakal gemessen) } \\
\text { in beiden Gruppen doppelt so groß wie im Au- } \\
\text { topsiegut, d.h., der Shunt hätte somit das } 4 \text { fache } \\
\text { Flusspotential }\end{array}$} \\
\hline $\begin{array}{l}\text { Follow-up (6 Monate) } \\
\text { Primärer Endpunkt } \\
\text { (s. o.) }\end{array}$ & 3 Patienten & $\begin{array}{l}3 \text { Patienten (somit } \\
\text { nicht erreicht) }\end{array}$ \\
\hline $\begin{array}{l}50 \% \text { ige Reduktion } \\
\text { der Migräneanfälle }\end{array}$ & $23 \%$ & $42 \%$ * \\
\hline $\begin{array}{l}\text { "Headache burden“ (Re- } \\
\text { duktion der Anfallsdauer } \\
\text { mal Anfallshäufigkeit) }\end{array}$ & $17 \%$ & $37 \%$ * \\
\hline Komplikationen & $\begin{array}{l}4 \text { Patienten (1 Patient: } \\
\text { lokale Blutung, } 2 \text { Patien- } \\
\text { ten: Blutungen unter } \\
\text { ASS + Clopidogrel, } \\
1 \text { Patient: Stamm- } \\
\text { hirninfarkt) }\end{array}$ & $\begin{array}{l}5 \text { Patienten (2 Patien- } \\
\text { ten: Tamponade oder } \\
\text { Perikarderguss, } 1 \text { Pa- } \\
\text { tient: lokale Blutung, } \\
1 \text { Patient Vorhofflim- } \\
\text { mern, } 1 \text { Patient, aty- } \\
\text { pischer Brustschmerz) }\end{array}$ \\
\hline
\end{tabular}

anfälle ursächlich auslösen. Vorangegangene retrospektive Studien bei Patienten mit Schlaganfällen zeigten, dass $22-57 \%$ an Migräne litten und dass die Prävalenz der Migräneanfälle mit der Größe des Shunts korrelierte. Nichtkontrollierte Interventionsstudien zeigten eine 65 - bis $90 \%$ ige Elimination oder Reduktion der Migräneanfälle durch einen perkutanen PFO-Verschluss. In der Studie sollte gezeigt werden, dass der perkutane, mechanische Verschluss eines PFO bei Patienten mit dokumentierter Migräne zu einer vollständigen Beschwerdefreiheit führt. Studienleiter war Peter Wilmshurst, Shrewsbury, UK.

\begin{tabular}{|c|c|c|}
\hline Klinischer primärer Endpunkt & $\mathrm{Ja}=3$, nein $=0$ & 3 \\
\hline Doppelblind (einschließlich Ärzten) & $\mathrm{Ja}=1$, nein $=0$ & 1 \\
\hline $\begin{array}{l}\text { Beobachtungsintervall für den } \\
\text { primären Endpunkt } \geq 6 \text { Monate }\end{array}$ & $\mathrm{Ja}=1$, nein $=0$ & 1 \\
\hline Multicenter (mindestens 3 Zentren) & $\mathrm{Ja}=1$, nein $=0$ & 1 \\
\hline $\begin{array}{l}\text { Externes und vom Steering } \\
\text { Comittee unabhängiges Clinical } \\
\text { Event Committee / DSMB (Daten- } \\
\text { sicherheit Monitoring Board) }\end{array}$ & $\mathrm{Ja}=1$, nein $=0$ & (?) \\
\hline Primärer Endpunkt erreicht & $\mathrm{Ja}=1$, nein $=0$ & 0 \\
\hline $\begin{array}{l}\text { Power von } \geq 80 \% \text { für den } \\
\text { primären Endpunkt erreicht }\end{array}$ & $\mathrm{Ja}=1$, nein $=0$ & 0 \\
\hline $\begin{array}{l}\text { Anteil des Follow-up der Patienten } \\
\text { für angiographischen primären } \\
\text { Endpunkt } \geq 80 \% \text { und } \geq 95 \% \text { für } \\
\text { klinischen primären Endpunkt }\end{array}$ & $\mathrm{Ja}=1$, nein $=0$ & 1 \\
\hline $\begin{array}{l}\text { Gesamt-EBM-Score } \\
\text { („Silber-Score“ für randomisierte Stuc }\end{array}$ & & 7 \\
\hline
\end{tabular}

Schlussfolgerung und Kommentar: Der primäre Endpunkt einer kompletten Elimination von Migräneanfällen durch perkutanen PFO-Verschluss wurde nicht erreicht. Die Reduktion der Häufigkeit und Dauer der Migräneanfälle war signifikant. Da noch nicht alle Parameter der Datenbank ausgewertet wurden, ist noch keine abschließende Aussage zu treffen. Die Studie besitzt wesentliche Limitationen: 1. Der Nachweis eines PFO wird standardmäßig durch eine transösophageale (TEE) und nicht durch eine transthorakale (TTE) Echokardiographie erbracht. Die TEE ist die wesentlich genauere Methode, insbesondere wenn die Größe des PFO bestimmt wird. In der Kardiologie würde niemand - auf einem TTE-Befund basierend - ein Okkludersystem implantieren. Ein transkranieller Doppler wurde in keinem Fall durchgeführt. 2. Die Hypothese, dass es spezifische Substanzen gibt, die bei der Lungenpassage normalerweise „entgiftet" werden, durch den Rechts-links-Shunt jedoch direkt in das ZNS gelangen, wo sie die Migräneanfälle ursächlich auslösen, ist spekulativ. 3. Die „Plazeboprozedur“ mit Vollnarkose und Inzision der Leiste erscheint im Hinblick auf die gravierenden Komplikationen sehr bedenklich. Derzeit kann die Implantation eines Okkludersystems bei PFO und dokumentierter Migräne außerhalb klinischer Studien nicht empfohlen werden.

Herz 2006;31:167

DOI 10.1007/s00059-006-2809-7 